\title{
Publisher Correction: Single-arm, open-label phase 2 trial of pembrolizumab in patients with leptomeningeal carcinomatosis
}

Priscilla K. Brastianos (1D, Eudocia Quant Lee, Justine V. Cohen, Sara M. Tolaney, Nancy U. Lin, Nancy Wang, Ugonma Chukwueke, Michael D. White (D), Naema Nayyar, Albert Kim, Christopher Alvarez-Breckenridge, lan Krop, Maura Keeley Mahar, Mia S. Bertalan, Brian Shaw, Joana L. Mora, Nathaniel Goss, Megha Subramanian, Lakshmi Nayak, Jorg Dietrich, Deborah A. Forst, Brian V. Nahed, Tracy T. Batchelor, Helen A. Shih, Elizabeth R. Gerstner, Beverly Moy, Donald Lawrence, Anita Giobbie-Hurder, Scott L. Carter ㄹ, Kevin Oh, Daniel P. Cahill and Ryan J. Sullivan (1)

Correction to: Nature Medicine https://doi.org/10.1038/s41591-020-0918-0, published online 2 June 2020.

In the version of this article initially published, the date published was given as 01 June 2020 . The correct date is 02 June 2020 . The error has been corrected in the HTML and PDF versions of the article.

Published online: 18 June 2020

https://doi.org/10.1038/s41591-020-0978-1

(๑) The Author(s), under exclusive licence to Springer Nature America, Inc. 2020

\section{Publisher Correction: Building an international consortium for tracking coronavirus health status}

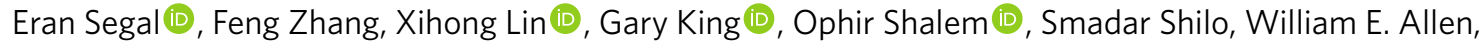
Faisal Alquaddoomi, Han Altae-Tran, Simon Anders (D), Ran Balicer, Tal Bauman, Ximena Bonilla (D), Gisel Booman (D), Andrew T. Chan (1), Ori Cohen, Silvano Coletti, Natalie Davidson, Yuval Dor, David A. Drew (D), Olivier Elemento, Georgina Evans, Phil Ewels (D), Joshua Gale, Amir Gavrieli, Benjamin Geiger, Yonatan H. Grad (D), Casey S. Greene (D), Iman Hajirasouliha, Roman Jerala (D), Andre Kahles, Olli Kallioniemi, Ayya Keshet, Ljupco Kocarev, Gregory Landua, Tomer Meir, Aline Muller, Long H. Nguyen, Matej Oresic (D), Svetlana Ovchinnikova, Hedi Peterson (D), Jana Prodanova, Jay Rajagopal, Gunnar Rätsch, Hagai Rossman, Johan Rung (D), Andrea Sboner, Alexandros Sigaras (1), Tim Spector (1D, Ron Steinherz, Irene Stevens, Jaak Vilo (D) and Paul Wilmes

Correction to: Nature Medicine https://doi.org/10.1038/s41591-020-0929-x, published online 2 June 2020.

In the version of this article initially published, the affiliation for authors David A. Drew and Long H. Nguyen $\left({ }^{14}\right.$ ETH Zurich, Department for Computer Science, Zurich, University Hospital Zurich, Medical Informatics, Zurich and SIB Swiss Institute of Bioinformatics, Zurich, Switzerland) was incorrect. The correct affiliation is ' ${ }^{16}$ Massachusetts General Hospital (MGH), Boston, MA, USA'. The error has been corrected in the HTML and PDF versions of the article.

Published online: 26 June 2020

https://doi.org/10.1038/s41591-020-0983-4

๑) Springer Nature America, Inc. 2020 